\title{
Colony-stimulating factor therapy and febrile neutropenia induced by chemotherapy: need for economical studies
}

\author{
T. Urban, B. Lebeau
}

Febrile neutropenia is the major dose-limiting toxicity of anticancer chemotherapy in small cell lung cancer (SCLC). Nadir and duration of neutropenia have been correlated to the risk of developing sepsis [1], increasing healthcare costs and deteriorating quality of life. Despite empirical broad-spectrum antibiotic therapy at the onset of fever and dose reduction of chemotherapy during subsequent cycles, neutropenia-induced mortality remains important, particularly in patients with extensive SCLC.

Multivariate analysis has shown that neutrophil recovery is a favourable prognostic factor in febrile neutropenia, independent of the use of antibiotic therapy [2]. The capacities of haematopoietic colony-stimulating factors (CSFs) to accelerate granular neutrophil recovery, as well as monocyte and eosinophil with granulocyte-macrophage CSFs (GM-CSFs), allows the hope of a reduction of haematological toxicity or an increased chemotherapy dose intensity. Primary prophylactic administration of granulocyte CSFs (G-CSFs), before the onset of neutropenia, reduced incidence of febrile neutropenia, length of hospitalization and antibiotic use subsequent to high-dose chemotherapy in SCLC patients [3, 4], whereas infectious mortality rate, response and survival rates were not improved. However, the cost-effectiveness of primary GCSF administration still remains controversial, with a high cost counterbalancing these benefits.

G-CSF therapy combined with antibiotic therapy, in which CSFs are initiated after onset of febrile neutropenia, avoiding their use in patients who might not experience febrile neutropenia and allowing the hope of a reduction of CSF costs, have been considered more recently. This strategy is based on the ability of CSF to activate neutrophils with an enhanced protective efficacy against infection. Neutrophils harvested from patients treated with G-CSF have been demonstrated to be functional and to control infections due to a range of different bacteria in animal models.

The 1994 American Society of Clinical Oncology (ASCO) practice guidelines [5] for the use of CSFs, stated that "therapeutic initiation of CSFs in addition to antibiotics at the onset of febrile neutropenia should be reserved for patients at high risk for septic complications, even though its benefits under these circumstances have not been yet proved". The updated ASCO recommendations [6], published in 1996, generated much discussion, and the conclusions concerning CSF therapy still remain unclear. In contrast, evaluating the patterns of use of the

Service de Pneumologie, Hôpital Saint-Antoine, Paris, France.

Correspondence: T. Urban, Service de Pneumologie, Hôpital SaintAntoine, 184 rue du Faubourg Saint-Antoine, 75012 Paris, France. Fax: 33149282283. haematopoietic CSFs in various clinical settings, BENETT et al. [7] found that $38.9 \%$ of physicians usually chose to use CSF therapy in febrile neutropenia, and $54.3 \%$ when pneumonia was present, while only $11.5 \%$ never used CSFs.

Seven randomized studies evaluating the effects of GCSF or GM-CSF therapy in patients who experienced febrile neutropenia at the time of inclusion have been reported since 1990 [8-14]. However, only four studies included $\geq 100$ patients, and the differential value of their conclusion in comparison to those of smaller studies must be taken into account. The difficulties in interpretation of reported data are emphasized by the heterogeneous chemotherapy regimens used in these studies.

In 1994, MAHER et al. [8] reported the largest randomized placebo-controlled study of G-CSF (filgrastim) therapy in 216 patients with nonmyeloid malignancies treated with various chemotherapy regimens. Patients with grade III/IV febrile neutropenia were treated with antibiotics and either continuous subcutaneous infusion high-dose $\left(12 \mu \mathrm{g} \cdot \mathrm{kg}^{-1} \cdot \mathrm{day}^{-1}\right)$ filgrastim $(\mathrm{n}=109)$ or placebo $(\mathrm{n}=107)$ until 4 days without fever and neutrophil count exceeding $0.5 \times 10^{9}$ cells $\cdot \mathrm{L}^{-1}$. Compared to placebo, filgrastim reduced the median duration of grade III/IV neutropenia and the time to resolution of febrile neutropenia (5 versus 6 days), whereas infectious mortality rates were not statistically different ( 7 versus $3 \%$ with placebo). This study was not designed to prospectively collect relevant costbenefit data, but the high-dose and 7 days of G-CSF therapy must be compared to the lower dosage $\left(5 \mu \mathrm{g} \cdot \mathrm{kg}^{-1}\right.$. day $^{-1}$ ) and median of 9 days of treatment with primary GCSF administration. However, the relative risk for prolonged hospitalization over 11 days was twice as high in the placebo group $(\mathrm{p}=0.02)$, and the small morbidity benefit for CSF was more evident in a subset of patients with documented infections [8].

In 1995, MAYORDOMO et al. [9] compared the use of GCSF $\left(5 \mu \mathrm{g} \cdot \mathrm{kg}^{-1} \cdot \mathrm{day}^{-1}\right)$ or GM-CSF $\left(5 \mu \mathrm{g} \cdot \mathrm{kg}^{-1} \cdot \mathrm{day}^{-1}\right)$ therapy to placebo in 121 non-leukaemic cancer patients with grade IV febrile neutropenia in combination with antibiotic therapy. Compared with placebo, CSFs reduced the median duration of grade IV neutropenia and hospital stay (5 versus 7 days), whereas infectious mortality rates were not different ( 7 versus $3 \%$, respectively). The median duration of G-CSF or GM-CSF use was 4 days. A trend toward a lower cost was observed in the CSF groups compared with the placebo group $(\mathrm{p}=0.11$ for $\mathrm{G}-\mathrm{CSF}$, and $\mathrm{p}=0.06$ for GM-CSF).

ANAISSIE et al. [10] compared GM-CSF therapy to placebo in 107 patients with nonmyeloid malignancies experiencing febrile neutropenia. Addition of GM-CSF to 
antibiotics did not improve the survival rate (both 93\%) and superinfections and subsequent infections were not significantly different. Moreover, side-effects were more common among patients treated with the GM-CSF. ANAISSIE et al. [10], therefore, did not recommend the routine administration of GM-CSF with antibiotics for patients with fever and neutropenia.

Comparing GM-CSF to placebo in 133 patients experiencing febrile neutropenia, VelLenga et al. [11] found a significant effect of GM-CSF on neutrophil recovery. In contrast, quality of life was significantly better in the placebo group and hospital costs appeared significantly higher for GM-CSF-treated patients.

Two other studies were less conclusive because of lower study populations $[12,13]$. Comparing GM-CSF $(n=28)$ to placebo $(n=30)$ in a paediatric population with febrile neutropenia, RIIKONEN et al. [12] found a significant effect in favour of GM-CSF on median duration of neutropenia, hospital stay, and antibiotic use in some patients. BIESMA et al. [13], comparing GM-CSF to placebo, found similar results in 30 nonmyeloid cancer patients with grade IV febrile neutropenia induced by various chemotherapy regimens.

Recently, RAVAUD et al. [14] have compared the use of GM-CSF $\left(5 \mu \mathrm{g} \cdot \mathrm{kg}^{-1} \cdot\right.$ day $\left.^{-1}\right)$ therapy to a control group without placebo, in 68 nonmyeloid cancer patients with febrile neutropenia after stratification of chemotherapies according to their risk of inducing a high or low frequency of febrile neutropenia. Interestingly, GM-CSF significantly shortened the duration of neutropenia and duration on antibiotics during hospitalization in patients receiving low-risk chemotherapy, but not in high-risk chemotherapy.

Consequently, data collected from the randomized patients with febrile neutropenia comparing G-CSF or GM-CSF to control patients provide little apparent benefit in morbidity or mortality for most patients. Data concerning length of hospital stay, an important component of the additional cost of chemotherapy-induced febrile neutropenia, do not support an evident cost benefit of CSF therapy [11]. Moreover, the mean mortality rate according to reported data was similar in both groups.

In conclusion, these data do not support the routine administration of granulocyte colony-stimulating factor or granulocyte-macrophage colony-stimulating factor with antibiotics for patients with chemotherapy-induced febrile neutropenia. Further studies with economical evaluation should be conducted to identify those patients most likely to benefit from colony-stimulating factor therapy, such as patients with persistent profound neutropenia and documented and persistent refractory infections. Small cell lung cancer patients treated with intensive chemotherapy are probably good candidates for such studies.

\section{References}

1. Radford JA, Ryder NDJ, Dodwell D, Anderson H, Thatcher N. Predicting septic complications of che- motherapy: an analysis of 381 patients treated for small cell lung cancer without dose reduction after major sepsis. Eur J Cancer 1993; 29A: 81-86.

2. Bodey GP, Fainstein V, Elting IS, et al. Beta-lactam regimens for the febrile neutropenic patient. Cancer 1990; 65: 9-16.

3. Crawford J, Ozer H, Stoller R, et al. Reduction by granulocyte colony-stimulating factor of fever and neutropenia induced by chemotherapy in patients with smallcell lung cancer. $N$ Engl J Med 1991; 325: 164-170.

4. Trillet-Lehoir V, Green J, Manegold C, et al. Recombinant granulocyte colony-stimulating factor reduces the infectious complications of cytotoxic chemotherapy. Eur J Cancer 1993; 29: 319-324.

5. Anonymous. American Society of Clinical Oncology recommendations for the use of hematopoietic colony-stimulating factors: evidence-based clinical practice guidelines. J Clin Oncol 1994; 12: 2471-2508.

6. Anonymous. Update of recommendations for the use of hematopoietic colony-stimulating factors: evidence-based clinical practice guidelines. J Clin Oncol 1996; 14: 19571960.

7. Bennett CL, Smith TJ, Weeks JC, et al. Use of hematopoietic colony-stimultaing factors: the American Society of Clinical Oncology survey. J Clin Oncol 1996; 14: 2511-2520.

8. Maher DW, Lieschke GJ, Green M, et al. Filgrastim in patients with chemotherapy-induced febrile neutropenia. A double-blind, placebo-controlled trial. Ann Intern Med 1994; 121: 492-501.

9. Mayordomo JI, Rivera F, Diaz-Puente M, et al. Improving treatment of chemotherapy-induced neutropenic fever by administration of colony-stimulating factors. $J$ Natl Cancer Inst 1995; 87: 803-808.

10. Anaissie EJ, Vartivarian S, Bodey GP, et al. Randomized comparison between antibiotics alone and antibiotics plus granulocyte-macrophage colony-stimulating factor (Escherichia coli-derived) in cancer patients with fever and neutropenia. Am J Med 1996; 100: 17-23.

11. Vellenga E, Uyl-de Groot CA, de Wit R, et al. Randomized placebo-controlled trial of granulocytemacrophage colony-stimulating factor in patients with chemotherapy-related febrile neutropenia. J Clin Oncol 1996; 14: 619-627.

12. Riikonen P, Saarinen UM, Makipernaa A, et al. Recombinant human granulocyte-macrophage colony-stimulating factor in the treatment of febrile neutropenia: a double-blind placebo-controlled study in children. Pediatr Infect Dis J 1994; 13: 197-202.

13. Biesma B, de Vries EG, Willemse PH, et al. Efficacy and tolerability of recombinant human granulocyte-macrophage colony-stimulating factor in patients with chemotherapy-related leukopenia and fever. Eur J Cancer 1990; 26: 932-936.

14. Ravaud A, Chevreau C, Cany L, et al. Granulocytemacrophage colony-stimulating factor in patients with neutropenic fever is potent after low-risk but not after high-risk neutropenic chemotherapy regimens: results of a randomized phase III trial. J Clin Oncol 1998; 16 : 2930-2936. 